\title{
Two New Saponins from Solidago gigantea
}

\author{
Angelika Lendl, Gottfried Reznicek* \\ Department of Pharmacognosy, University of Vienna, Althanstrasse 14, \\ A-1090 Vienna, Austria
}

\begin{abstract}
Two new triterpenoid saponins, named giganteasaponin 5 and giganteasaponin 6 , were isolated from the aboveground parts of Solidago gigantea and were defined to be 3-O-[ $\beta$-D-Apio-D-furanosyl-( $1 \rightarrow 3)$ - $\beta$-D-glucopyranosyl-( $1 \rightarrow 3)$ - $\beta$-D-glucopyranosyl]-28-O \{ $\alpha$-L-rhamnopyranosyl-( $1 \rightarrow 3)-\beta$-D-xylopyranosyl-( $1 \rightarrow 4)$ - [ $\beta$-D-xylo-

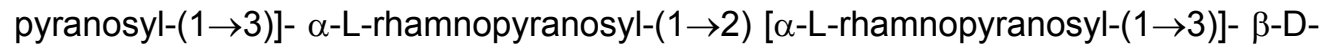
xylopyranosyl\}-Bayogenin respectively $3-O-[\beta-D-A p i o-D-f u r a n o s y l-(1 \rightarrow 3)-\beta-D-$ gluco-pyranosyl-( $1 \rightarrow 3)-\beta$-D-glucopyranosyl]-28-O $\{\beta$-D-galactopyranosyl-( $1 \rightarrow 2)-\alpha-$ L-rhamnopyranosyl-( $1 \rightarrow 3)-\quad \beta$-D-xylopyranosyl-(1 $\rightarrow 4)-\quad[\beta$-D-xylopyranosyl-( $1 \rightarrow 3)]-$ $\alpha$-L-rhamnopyranosyl-(1 $\rightarrow 2) \quad[\alpha-L-r h a m n o p y r a n o s y l-(1 \rightarrow 3)]-\quad \beta$-D-xylopyranosyl\}Bayogenin. The structures were established on the basis of chemical degradation, extensive MS studies and NMR investigations as well.
\end{abstract}

\section{Keywords}

Triterpen, saponin, giganteasaponin, Solidago gigantea

\section{Introduction}

In previous publications we reported on the isolation and structure elucidation of the main saponins (giganteasaponins 1-4, Figure 1) from Solidago gigantea [1-5]. Investigating the saponin content and composition of Solidago gigantea from different locations using HPLC [6] we found two additional unknown saponins (giganteasaponin 5 and 6 ) representing sometimes up to $20 \%$ of the total saponin 


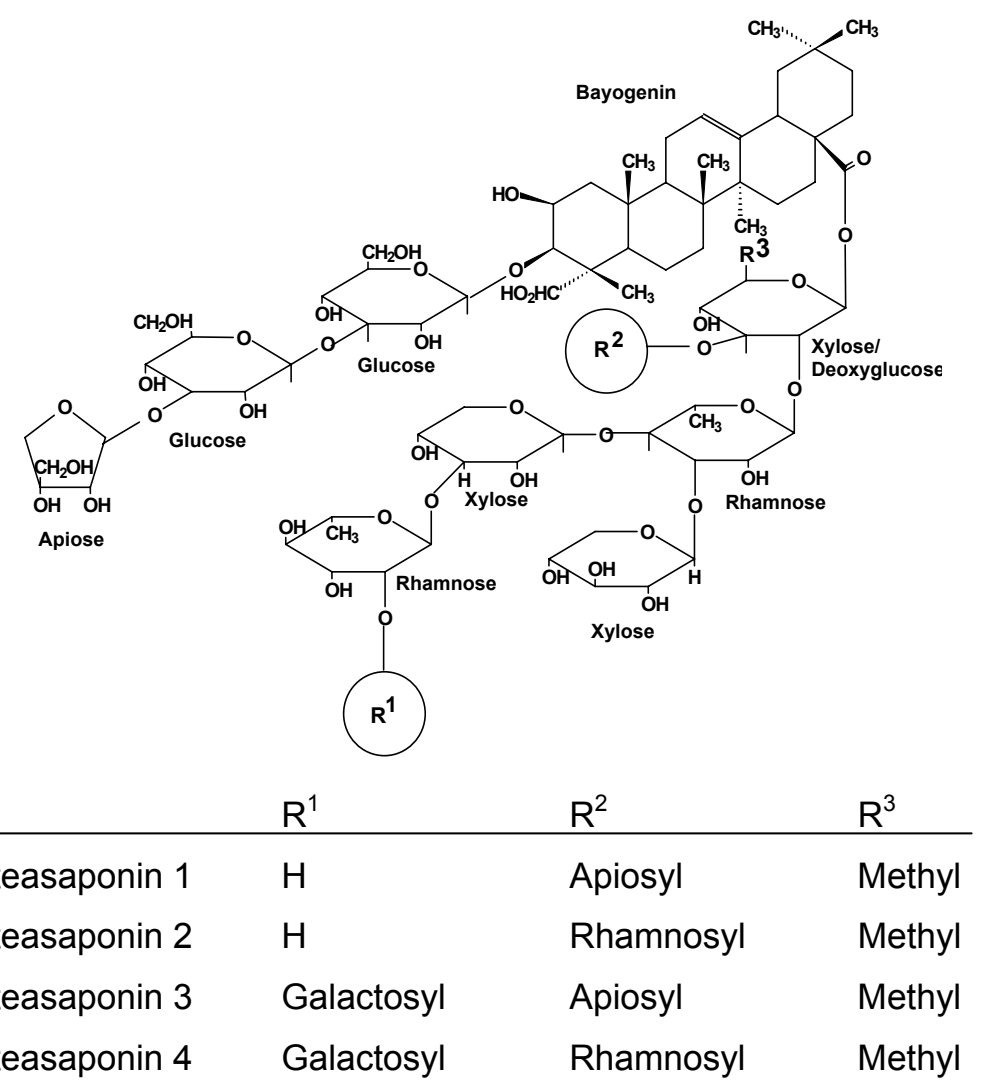

Figure 1. Structures of the known giganteasaponins from Solidago gigantea

content. The behaviour of giganteasaponin 5 and 6 at the HPLC analysis was similar to that of the other saponins from $S$. gigantea $[6,7]$ : pairs of saponins (giganteasaponins 1 and 3 respectively giganteasaponins 2 and 4) cannot be separated on reversed phase HPLC, they coelute in one peak and can only be separated using polar stationary phases like silica or polar RP-phases [7]. These pairs of saponins show an identical structure exept an additional terminal galactose in giganteasaponin 3 respectively gigantaesaponin 4 . Since giganteasaponin 5 and 6 coelute slightly before the other pairs of saponins on RP-HPLC it could be suggested that these compounds represent an analogous pair of saponins with 
similar polarity and identical structure except an additional galactose. In order to verify this assumption the aim of this study was the isolation and structure elucidation of giganteasaponin 5 and 6 .

\section{Results}

For the isolation of the saponins from Solidago gigantea a methanol-water extract $(80 \%)$ of dried aboveground parts was prepared. This extract was further separated by column chromatography over Sephadex LH-20 and the fractions were analysed by TLC to detect the saponins. The saponin containing fractions were further purified by SPE with C18 cartridges followed by a separation step using praparative HPLC on RP-8 with methanol-water as mobile phase. The coeluting giganteasaponins 5 and 6 could be separated by preparative TLC on silica and a final purification by preparative HPLC on RP-8 resulted in the single giganteasaponin 5 respectively 6 with high purity.

The spectral features, physicochemical properties, chromatographic behaviour and the hemolytic activity confirmed giganteasaponin 5 and 6 to be triterpenoid saponins. After enzymatic hydrolysis of the single saponins bayogenin could be detected as aglycon on TLC with an authentic reference sample. Hydrolysis of giganteasaponin 5 yielded apiose, glucose, rhamnose and xylose that could be separated and identified by HPIC with authentic reference samples. After trimethylsilylation the sugars were separated and identified by GLC [8] resp. GCMS and the ratio was determined to be approximatively 1:2:3:3. Hydrolysis of giganteasaponin 6 yielded similar results, above that an additional galactose could be identified. These results confirmed the previous suggestions giganteasaponins 5 and 6 to be a pair of saponins with identical sapogenins and identical sugar moieties exept an additional galactose in giganteasaponin 6 . To obtain the absolute configurations of the single carbohydrates we followed well established procedures [9] and found D-apiose, D-glucose, L-rhamose as well as D-xylose to be present in gigantaesaponin 5 , as expected, giganteasaponin 6 showed the same sugars and an additional D-galactose. 
The ESI-mass spectrum of giganteasaponin 5 showed a quasimolecular ion of $\mathrm{m} / \mathrm{z} 1777[\mathrm{M}-\mathrm{H}]^{-}$indicating a molecular weight of 1778 like giganteasaponin 1 (Figure 1). This result was confirmed by LSI-MS giving the same quasimolecular ion. The sapogenin bayogenin was found at $\mathrm{m} / \mathrm{z} 487$ and the typical series $\mathrm{m} / \mathrm{z} 487$ $\mathrm{m} / \mathrm{z} 649-\mathrm{m} / \mathrm{z} 811-\mathrm{m} / \mathrm{z} 943$, well known from the other giganteasaponins indicating the sugar moiety attached to $\mathrm{C}-3$ of bayogenin consisting of glucose, glucose and a terminal apiose (Figure 1).

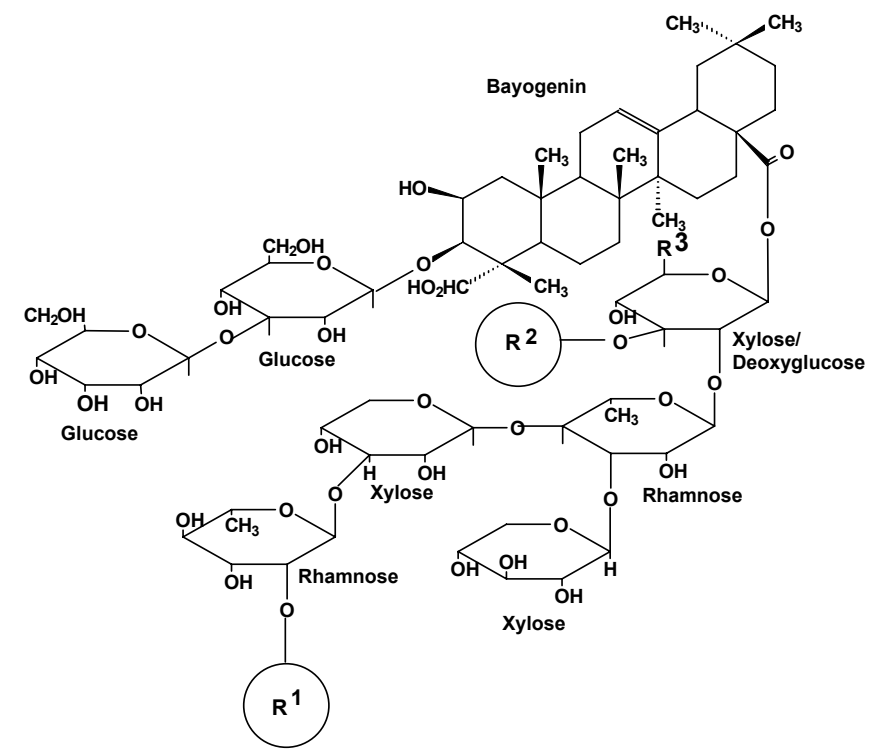

\begin{tabular}{llll} 
& R1 & R2 & R3 \\
\hline Canadensissaponin 1 & H & Apiosyl & Methyl \\
Canadensissaponin 2 & H & Apiosyl & H \\
Canadensissaponin 3 & H & Rhamnosyl & Methyl \\
Canadensissaponin 4 & H & Rhamnosyl & H \\
Canadensissaponin 5 & Galactosyl & Apiosyl & Methyl \\
Canadensissaponin 6 & Galactosyl & Apiosyl & H \\
Canadensissaponin 7 & Galactosyl & Rhamnosyl & Methyl \\
Canadensissaponin 8 & Galactosyl & Rhamnosyl & H
\end{tabular}

Figure 2. Structures of the known canadensissaponins from Solidago canadensis 
This sequence could also be observed starting from the quasimolecular ion $(\mathrm{m} / \mathrm{z}$ 1777- $\mathrm{m} / \mathrm{z}$ 1645- m/z 1483- $\mathrm{m} / \mathrm{z}$ 1321). Concerning the sugar moiety at C-28 we found sequences like m/z 1777-m/z 1631-m/z 1499-m/z 1367m that could be explained to be $[\mathrm{M}-\mathrm{H}]^{-}$-desoxyhexose (146)-pentose (132)-pentose (132) as well as $\mathrm{m} / \mathrm{z}$ 833-m/z 701-m/z 555, suggested to be a hexasaccharide-pentose (132)desoxyhexose (146). In fact the fragmentation of the acylglycosidic linked sugar moiety was similar but not the same to that of giganteasaponin 1 , indicating that there are differences and we found a perfect match with the fragmentation of the respective sugar moiety of canadensissaponin 4 from Solidago canadensis [10-12] (Figure 2), where a xylose is directly linked to C-28 instead of a desoxyglucose at giganteasaponin 1 . These results confirmed our sugar analyses, where no desoxyglucose could be found. These results could be confirmed by NMR studies, the spectrum was very close to that of giganteasaponin 2 [2], but again no desoxyglucose could be found and instead of this sugar a xylose could be identified. Using various NMR techniques the structure of giganteasaponin 5 could clearly be identified (Figure 3).

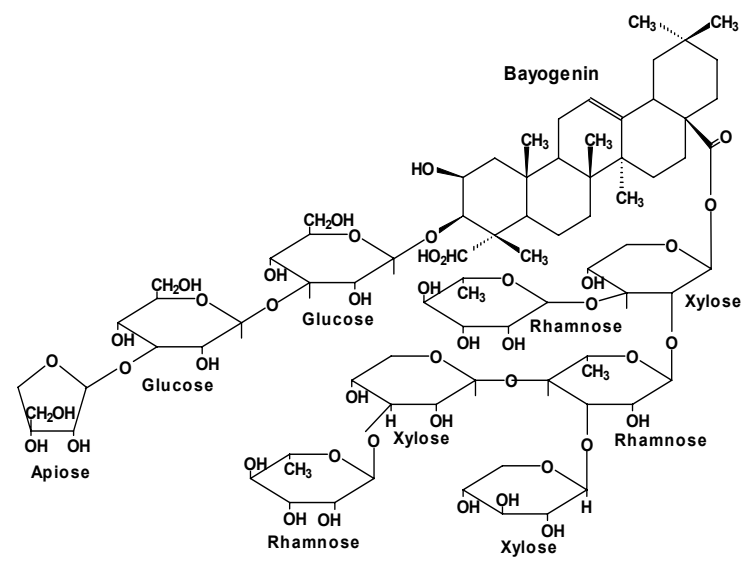

Figure 3. Structure of giganteasaponin 5

The ESI-mass spectrum of giganteasaponin 6 showed a quasimolecular ion of $\mathrm{m} / \mathrm{z} 1939[\mathrm{M}-\mathrm{H}]^{-}$indicating a molecular weight of 1940 like giganteasaponin 3 
(figure 1). This confirmed our prevoious suggestions that giganteasaponin 6 shows the same structure like giganteasaponin 5 exept an additional galactose. This result was confirmed by LSI-MS giving the same quasimolecular ion. The sapogenin bayogenin was again found at $\mathrm{m} / \mathrm{z} 487$ and the typical series $\mathrm{m} / \mathrm{z} 487-\mathrm{m} / \mathrm{z} 649-\mathrm{m} / \mathrm{z}$ $811-\mathrm{m} / \mathrm{z}$ 943, as well as m/z 1777-m/z 1645-m/z 1483-m/z 1321 could be observed indicating the sugar moiety attached to $\mathrm{C}-3$ of bayogenin consisting of glucose, glucose and a terminal apiose (Figure 1). Concerning the sugar moiety at C-28 a terminal hexose could be found in contrast to giganteasaponin 5 and the fragmentation pattern was found to be the same as the respective partial structure of canadensissaponin 8 [10-12] (Figure 2). Therefore it could be assumed that the structure of giganteasaponin 6 is identical with that of giganteasaponin 5 exept an additional terminal galactose. These results could be confirmed by NMR studies, the spectrum was very close to that of giganteasaponin 4 [2], but again no desoxyglucose could be found and instead of this sugar a xylose could be identified. Using various NMR techniques the structure of giganteasaponin 6 could be unequivocally identified (Figure 4).

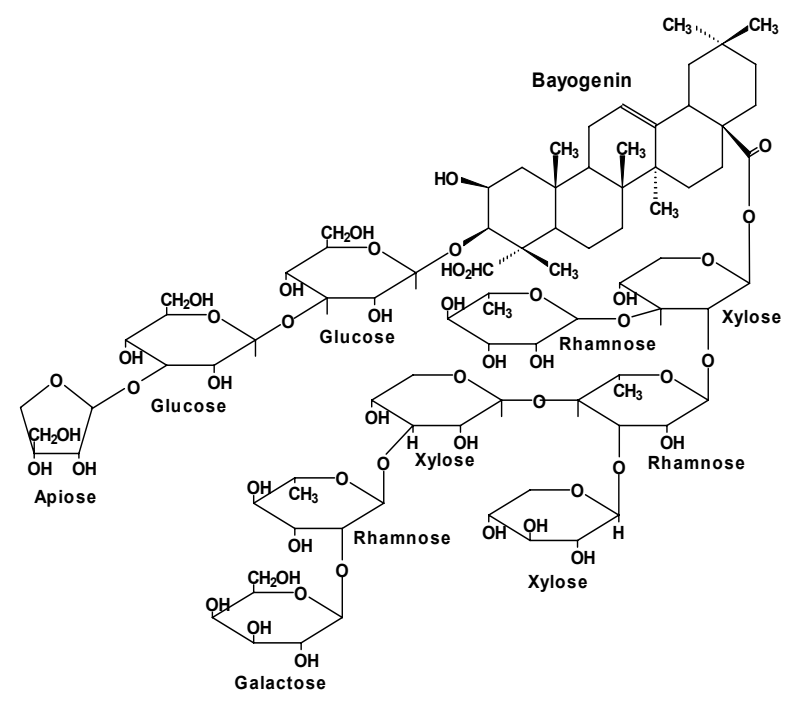

Figure 4. Structure of giganteasaponin 6 


\section{Experimental}

\section{General Experimental Procedures.}

Melting points were measured using a Reichert Heiztischmikroskop $\mathrm{RCH}$, LSI-MS was carried out on a Finnigan MAT 8500 (matrix glycerol, negative mode, Cs) and ESI-MS were recorded with PE-Sciex API 150 EX mass spectrometer (ESI, negative mode). ${ }^{1} \mathrm{H}$ and ${ }^{13} \mathrm{C}$ NMR were recorded on a $500 \mathrm{MHz}$ Bruker AM 500 spectrometer referring to TMS. TLC was used for saponin fraction control either on silica $60 \mathrm{~F}_{254}$ Merck precoated plates, mobile phase $\mathrm{CHCl}_{3}: \mathrm{CH}_{3} \mathrm{OH}$ :aqueous $\mathrm{HCOOH}(1 \%)=6: 5: 1$, or on HPTLC RP-8 $\mathrm{F}_{254}$ MERCK, mobile phase $\mathrm{CH}_{3} \mathrm{OH}$ :aqueous $\mathrm{HCOOH}(1 \%)=65: 35$, detection by spraying with anisaldehydesulfuric acid reagent (saponins give green spots). TLC for analysing the sapogenins was carried out on silica $60 \mathrm{~F}_{254}$ Merck precoated plates, mobile phase $\mathrm{CHCl}_{3}: \mathrm{CH}_{3} \mathrm{OH}=95: 5$, detection by spraying with anisaldehyde-sulfuric acid reagent (sapogenins give blue-grey spots). Sephadex LH-20 was used for column chromatography under normal pressure and solid phase extraction (SPE) was carried out with cartridges Inchrom Clean-UP C18, $5000 \mathrm{mg}$. Preparative HPLC was carried out using a Shimadzu preparative HPLC-system on a reversed phase column (LiChrosorb RP-8, $7 \mu \mathrm{m}, 25 \mathrm{~mm}$ i.d. x $250 \mathrm{~mm}$, detection UV $205 \mathrm{~nm}$ or split to evaporative light scattering detector Sedex 75). For carbohydrate analyses a Dionex 4500 HPAEC system was used (Carbo Pac PA-100, 4 x 250 mm, precolumn $4 \times 40 \mathrm{~mm}$, detection with PAD, mobile phase gradient $100 \mathrm{mM} \mathrm{NaOH}$ to $1 \mathrm{mM} \mathrm{NaOAc}$ in $100 \mathrm{mM} \mathrm{NaOH}$ ). Sugars were also analysed after trimethylsilylation using a Shimadzu QP2010 GCMS-system (capillary column Macherey-Nagel SE$54 \mathrm{CB}, 50 \mathrm{~m} \times 0,25 \mathrm{~mm}$ i.d., film $0,45 \mu \mathrm{m}$, injector $270^{\circ} \mathrm{C}$, oven $100-250^{\circ}$, rate $6 \%$ min., mobile phase He 5.0 , detector EIMS, $250^{\circ} \mathrm{C}, 70 \mathrm{eV}$ ).

\section{Plant material}

Solidago gigantea was collected in the "Konsumwald", Auhof, Vienna, Austria in August 1996. Voucher specimen are deposited in the herbarium of the Department of Pharmacognosy, University of Vienna. 


\section{Extraction and Isolation}

$700 \mathrm{~g}$ of the aboveground parts were dried and grinded and extracted exhaustively 3 times with 3 । 80\% methanol. The solvent was evaporated (residue $230 \mathrm{~g}$ ) and $20 \mathrm{~g}$ of the extract were separated by column chromatography on Sephadex LH-20 using methanol as mobile phase. Further purification was achieved by solid phase extraction (SPE) with cartridges Inchrom Clean-UP C18, $5000 \mathrm{mg}$, using methanol-water (40:60) and chloroform to purifiy the extract and methanol to elute the saponins. The saponins could be separated by repeated preparative HPLC on reversed phase (RP-8) using isocratic methanol-water (60:40) as mobile phase. The separation of giganteasaponin 5 and 6 could be achieved by repeated preparative TLC on silica 60 with chloroform-methanol-water $(6: 4: 1)$ as mobile phase. A final purification step with preparative HPLC on RP-8 using again methanol-water (60:40) as mobile phase gave giganteasaponin $5(17,66 \mathrm{mg})$ as well as giganteasaponin $6(15,45 \mathrm{mg})$ with high purity.

\section{Giganteasaponin 5}

3-O- $\quad[\beta$-D-Apio-D-furanosyl-(1 $\rightarrow 3)-\quad \beta$-D-glucopyranosyl-(1 $\rightarrow 3)-\quad \beta$-D-gluco-

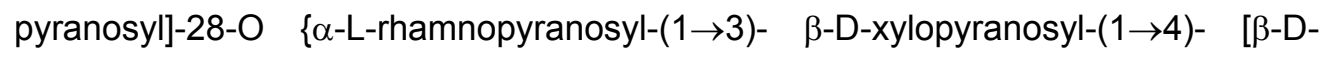
xylopyranosyl-( $1 \rightarrow 3)]-\quad \alpha$-L-rhamnopyranosyl-(1 $\rightarrow 2)[\alpha-L-r h a m n o p y r a n o s y l-(1 \rightarrow 3)]-$ $\beta$-D-xylopyranosyl\}-Bayogenin. White amorphous solid, $\mathrm{mp} 230-234^{\circ} \mathrm{C}$ (uncorr.). LSI-MS: m/z 1777 [M-H]', 1645, 1631, 1499, 1483, 1367, 1321, 943, 833, 811, 701, $649,555,487$.

\section{Giganteasaponin 6}

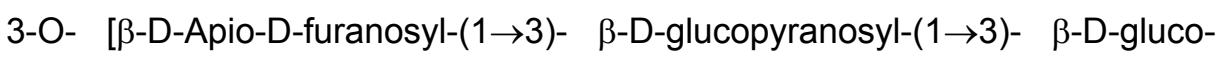

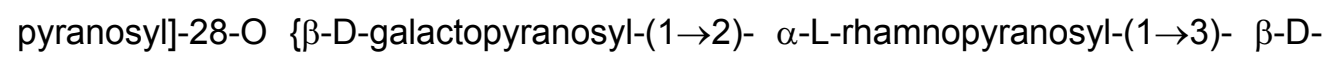
xylopyranosyl-( $1 \rightarrow 4)$ - [ $\beta$-D-xylopyranosyl-( $1 \rightarrow 3)]$ - $\alpha$-L-rhamnopyranosyl-( $1 \rightarrow 2)[\alpha-L-$ rhamnopyranosyl-(1 $\rightarrow 3)]$ - $\beta$-D-xylopyranosyl\}-Bayogenin. White amorphous solid, mp 253-258 ${ }^{\circ} \mathrm{C}$ (uncorr.), LSI-MS: m/z 1939 [M-H]', 1807, 1777, 1645, 1631, 1513, 1499, 1483, 1465, 1367, 995, 943, 863, 811, 717, 701, 649, 555, 487. 


\section{Discussion}

The main saponins from Solidago gigantea [1-5] and Solidago canadensis [10-12] are very similar, whereas the saponins from Solidago virgaurea have the same aglycone bayogenin but show different structures with less carbohydrates [13]. All saponins from $S$. canadensis have attached a disaccharide to C-3, consisting of two glucoses, in contrast to that the saponins from $S$. gigantea show a trisaccharide with two glucoses and an apiose at this position. Concerning the acylglycosidic sugar moiety at $\mathrm{C}-28$, the saponins from $\mathrm{S}$. gigantea and $\mathrm{S}$. canadensis are quite similar with six ore seven sugars. Taking a closer look to the saponins from the latter two species, pairs of saponins are found (giganteasaponins $1+3,2+4,5+6$ respectively canadensissaponins $1+5,2+6,3+7$ as well as $4+8$ ) with the identical structure exept an additional terminal galactose.

\section{References}

[1] Reznicek G, Jurenitsch J, Michl G, Haslinger E.

The first structurally confirmed saponin from Solidago gigantea: structure elucidation by modern NMR techniques.

Tetrahedron Letters 1989; 30: 4097-4100.

[2] Reznicek G, Jurenitsch J, Kubelka W, Michl G, Korhammer S, Haslinger E. Isolierung und Struktur der vier Hauptsaponine aus Solidago gigantea var. serotina.

Liebigs Ann. Chem. 1990; 989-994.

[3] Reznicek G., Jurenitsch J., Michl G. und Haslinger E.

Structure Elucitation of a Saponin from Solidago gigantea.

Bull. Magn. Resonance 1989; 11: 402.

[4] Reznicek G., Jurenitsch J., Michl G., Haslinger E., Hiller K, Kubelka W.

Structure of Two New Saponins from Solidago gigantea.

Planta medica 1989; 55: 623.

[5] Reznicek G., Jurenitsch J., Kubelka W., Michl G, Haslinger E. Struktur eines weiteren Hauptsaponins aus Solidago gigantea.

Sci.Pharm. 1989; 57:206.

[6] Reznicek G., Freiler M., Schader M., Schmidt U.

Determination of the content and the composition of the main saponins from Solidago gigantea AIT. using high performance liquid chromatography.

J. Chromatogr. 1996; 293: 133-137. 
[7] Reznicek G, Jurenitsch J, Kubelka W.

Analysis of the saponins from Solidagio gigantea and solidago canadensis by HPLC-MS

Joint meeting "2000 years of Natural Product Research", July 26-30, 1999, Amsterdam, Netherlands.

Book of Abtracts, Nr. 491

[8] Kopp, B.; Jurenitsch, J.; Kubelka, W.

Gaschromatographische Erfassung von 6-Desoxyhexosen, Pentosen und Hexosen aus herzwirksamen Glykosiden

J. Chrom 1981; 210: 291-299.

[9] Reznicek, G.; Susman, O., Böhm, K.

Bestimmung der Reihenzugehörigkeit von Monosacchariden aus pflanzlichen Glykosiden mittel GC-MS.

Sci. Pharm. 1993; 61: 35-45.

[10] Reznicek G, Jurenitsch J., Kubelka W., Korhammer S., Haslinger E. und Hiller K.

The First Spectroscopically Confirmed Saponins from Solidago Canadensis. Planta medica 1990; 56: 554.

[11] Reznicek G, Jurenitsch J, Plasun M, Korhammer S, Haslinger E, Hiller K, Kubelka W.

Four Major Saponins from Solidago canadensis.

Phytochemistry 1991; 30: 1629-1633.

[12] Reznicek G., Jurenitsch J., Freiler M., Korhammer S., Haslinger E., Hiller K, Kubelka W.

Isolierung und Struktur weiterer neuer Saponine aus Solidago canadensis.

Planta med. 1992; 58: 94-98.

[13] Hiller K., Bader G., Reznicek G., Jurenitsch J, Kubelka W.

Die Hauptsaponine der arzneilich genutzten Arten der Gattung Solidago.

Pharmazie 1991; 46: 405-408 . 